\title{
Local Binary Pattern Family Descriptors for Texture Classification
}

\author{
E. Jebamalar Leavline, D. Asir Antony Gnana Singh \\ Anna University, BIT Campus, Tiruchirappalli - 620024. \\ Email: \{jebilee@gmail.com, asirantony\}@gmail.com \\ P. Maheswari \\ K. Ramakrishnan College of Technology, Samayapuram, Tiruchirappalli-621 112. \\ Email: pmaheswarir@gmail.com
}

Received: 06 June 2018; Accepted: 13 August 2018; Published: 08 October 2018

\begin{abstract}
Texture classification is widely employed in many computer vision and pattern recognition applications. Texture classification is performed in two phases namely feature extraction and classification. Several feature extraction methods and feature descriptors have been proposed and local binary pattern (LBP) has attained much attraction due to their simplicity and ease of computation. Several variants of LBP have been proposed in literature. This paper presents a performance evaluation of LBP based feature descriptors namely LBP, uniform LBP (ULBP), LBP variance (LBPV), LBP Fourier histogram, rotated LBP (RLBP) and dominant rotation invariant LBP (DRLBP). For performance evaluation, nearest neighbor classifier is employed. The benchmark OUTEX texture database is used for performance evaluation in terms of classification accuracy and runtime.
\end{abstract}

Index Terms-Local binary pattern, texture classification, rotation invariance, Fourier histogram.

\section{INTRODUCTION}

Texture defines the feel or appearance of a surface of an object. Texture analysis is a major area of pattern recognition and computer vision. There are four major functions of texture analysis, which includes feature extraction, texture discrimination, texture classification, and extracting shape from texture [1]. This paper deals with texture classification using LBP family descriptors. LBP is a simple and efficient feature descriptor that is used in many applications in computer vision and pattern recognition. Many researchers have successfully employed LBP and its variants as face descriptor for face recognition [2]. Nguyen et al. presented object detection using non-redundant local binary patterns [3]. Trefný et al. proposed the extended set of local binary patterns for rapid object detection [4]. Heikkilä et al. used LBP features for description of interest regions [5].

However, texture classification using LBP features has attracted many researchers. This is due to the fact that texture features are localized and LBP can efficiently capture these local features in both grayscale and colour images [6]. Several enhancements to the original LBP have been proposed in the literature. These LBP variants can be classified broadly into three categories. The first category mainly focuses on uniformity of LBP pattern [7]. The class of LBP variants with rotation invariance capability falls under second category [8]. The third category of LBP variants employs certain feature selection mechanism to extract dominant LBP features in turn reduces the dimensionality of the feature space [9].

Texture classification has four major phases as shown in Fig. 1. Initially, the texture image databases are collected and the features are extracted from each texture image in the database. For classification, a subset of the complete database is used as training set and the complementary subset is used to test the classification model. For simplicity, the nearest neighbor classifier is employed in this work. Finally the performance of texture classification is evaluated. This paper presents the performance evaluation of LBP variants such as simple LBP, ULBP, LBP-HF, LBPV, RLBP, and DRLBP. The performance of these LBP variants is evaluated on OUTEX database [10] in terms of classification accuracy, error rate, sensitivity and specificity for various LBP parameters. The rest of the paper is organized as follows. Section 2 describes the texture feature classification using LBP variants. Section 3 discusses the experimental results and Section 4 concludes the paper. 


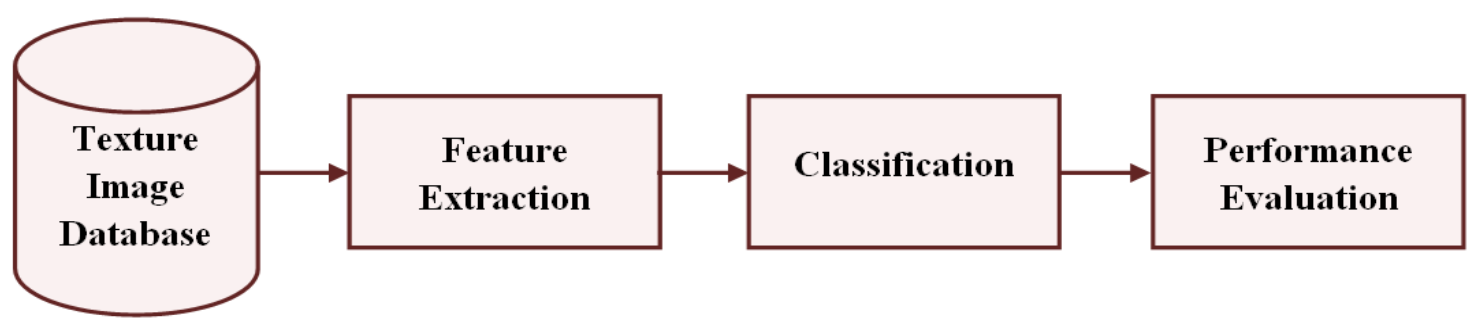

Fig.1. General texture classification framework

\section{LBP FAMILY DESCRIPTORS FOR TEXTURE CLASSIFICATION}

\section{A. Local Binary Pattern (LBP)}

Local binary pattern is derived from the relationship of a particular pixel with its neighbors. It first calculates the difference between each neighbor pixel with the reference pixel. A thresholding operator is applied considering the gray level of the reference pixel as threshold as in Equation (1). $\mathrm{P}$ is the number of neighbors considered and $\mathrm{R}$ is the radius of neighborhood. This results in a binary representation with 8 bits with each bit

representing a neighbor. The corresponding decimal value is the LBP of the reference pixel as illustrated in Fig. 1. Repeat the same process for steps in the image and compute histogram of the LBP values which is generally used as a feature vector. The dimensionality of LBP feature vector is 256 [11].

$$
\begin{gathered}
L B P_{P, R}=\sum_{p=0}^{P-1} T\left(g_{p}-g_{c}\right) 2^{p} \\
\because T(x)= \begin{cases}1, & \text { if } x \geq 0 \\
0, & \text { else }\end{cases}
\end{gathered}
$$

\begin{tabular}{|c|c|c|}
\hline 30 & 25 & 15 \\
\hline 10 & 20 & $\mathbf{1 0}$ \\
\hline 15 & 25 & 30 \\
\hline
\end{tabular}

Sample Image

\begin{tabular}{|c|c|c|}
\hline 10 & 5 & -5 \\
\hline-10 & & $\mathbf{- 1 0}$ \\
\hline-5 & 5 & 10 \\
\hline
\end{tabular}

$\left(\mathrm{g}_{\mathrm{p}}-\mathrm{g}_{\mathrm{c}}\right)$

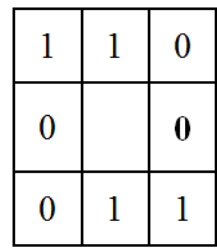

$\mathrm{T}\left(\mathrm{g}_{\mathrm{p}}-\mathrm{g}_{\mathrm{c}}\right)$

$$
01100110_{2}=102_{10}
$$

Fig.2. Illustration of LBP Calculation (position $p=0$ is shown in boldface)

\section{B. Uniform Local Binary Pattern (ULBP)}

Uniform LBP is derived from LBP calculated as shown in Equation (1) and by selecting only the uniform patterns [12]. A local binary pattern is said to be uniform if there is only maximum two transitions from $0-1$ or $1-0$. Eg: 01110000,11111100 . The major advantage of ULBP is the reduction in dimensionality and reduced computation complexity $[8,12]$.

\section{Local Binary Pattern - Histogram Fourier (LBP-HF)}

This is computed from discrete Fourier transform of LBP histogram. It is constructed globally for the entire region of interest. First the non-invariant LBP is constructed over the entire image. Then rotationally invariant features are constructed from the Fourier transform of LBP histogram. Since only uniform patterns are considered, the dimensionality of the feature vector is significantly reduced [8].

\section{Local Binary Pattern Variance (LBPV)}

The simple LBP describes the local spatial pattern and a variance operator is often used to represent the local contrast of the image block considered. LBPV descriptor proposed in [13] is a combined description of both the local spatial pattern and contrast. However, the local variance is used to assign adaptive weight to each LBP as in Equation (2) and (3).

$$
\begin{aligned}
& L B P V_{P, R}(k)=\sum_{i=1}^{N} \sum_{j=1}^{M} W\left(L B P_{P, R}(i, j), k\right), \quad k \in[0, K] \\
& W\left(L B P_{P, R}(i, j), k\right)= \begin{cases}\operatorname{VAR}_{P, R}(i, j), & L B P_{P, R}(i, j)=k \\
0, & \text { else }\end{cases}
\end{aligned}
$$

\section{E. Rotated Local Binary Pattern (RLBP)}

RLBP first computes the simple LBP and assigns the binary weights based on the dominant direction. The dominant direction is calculated as the index of maximum gray level change with respect to the reference pixel as in Equation (4). The dominant direction is given the least weight as in Equation (5) where T(.) is the same as in Equation (1). This is true even if the image is rotated [14].

$$
\begin{aligned}
& D=\underset{p \in(0,1,--P-1)}{\arg \max }\left|g_{p}-g_{c}\right| \\
& R L B P=\sum_{p=0}^{P-1} T\left(g_{p}-g_{c}\right) \cdot 2^{\bmod (p-D, P)}
\end{aligned}
$$




\section{F. Dominant Rotation-Invariant Local Binary Pattern (DRLBP)}

DRLBP features proposed in [15] are extracted based on RLBP. Unlike many LBP variants, DRLBP retains the non-uniform patterns too for exploring the most important discriminative features of the local image patch This is achieved by utilizing only the most frequently occurring patterns for classification irrespective of their uniformity. Hence, the dimensionality is significantly reduced while preserving the discriminative power of the features. However, the dimensionality is not constant as it depends on the threshold frequency and the inherent image characteristics itself.

\section{EXPERIMENTAL RESULTS AND DISCUSSION}

This section presents the details of dataset, performance evaluation metrics, experimental setup and procedure. In addition, the experimental results and discussion are presented.

\section{A. Dataset}

The data are collected from OUTEX texture database. It has 16 texture databases with distinct rotation in .ras or .bmp format. For our experiments, we collected 7 databases as listed in Table 1.

Table 1. Details of Databases

\begin{tabular}{|l|l|l|l|l|l|l|l|}
\hline $\begin{array}{l}\text { Database } \\
\text { OUTEX }\end{array}$ & $\begin{array}{l}\text { No. of } \\
\text { Images }\end{array}$ & $\begin{array}{l}\text { Training } \\
\text { Set }\end{array}$ & Test Set & Image Size & No. of Classes & Rotations & $\begin{array}{l}\text { Image File } \\
\text { Format }\end{array}$ \\
\hline TC -0010 & 4320 & 480 & 3840 & $128 \times 128$ & 24 & $\begin{array}{l}00,05,10,15, \\
30,45,60,75,90\end{array}$ & RAS \\
\hline TC -0011 & 960 & 480 & 480 & $128 \times 128$ & 24 & $\begin{array}{l}00,05,10,15, \\
30,45,60,75,90\end{array}$ & RAS \\
\hline TC -0012 & 9120 & 480 & 4320 & $128 \times 128$ & 24 & 00 & 00 \\
\hline TC -0013 & 1360 & 680 & 680 & $128 \times 128$ & 68 & 00 & RAS \\
\hline TC -0014 & 4080 & 680 & 1360 & $128 \times 128$ & 68 & $00,05,10,15$, \\
\hline TC -0020 & 12240 & 1360 & 10880 & $128 \times 128$ & 68 & $\begin{array}{l}00,05,10,15, \\
30,45,60,75,90\end{array}$ & BMP \\
\hline TC -0030 & 12240 & 1360 & 10880 & $128 \times 128$ & 68 & BMP \\
\hline
\end{tabular}

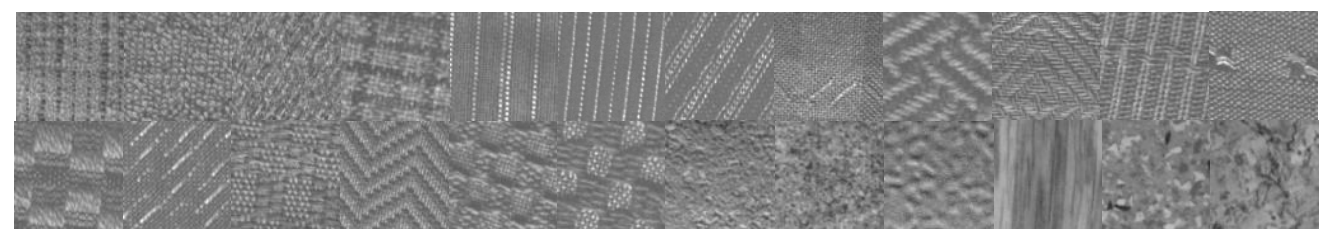

Fig.3. Sample texture images from each class in Outex 10 database

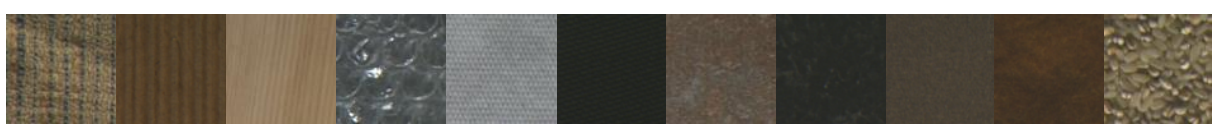

Fig.4. Sample texture images from each major class in Outex13 database

All the 7 databases have texture images of size $128 \times 128$. Each database is split into training and test set as shown in Table 1. The OUTEX - 10, OUTEX -11 and OUTEX -12 databases have 24 classes with samples of canvas, tile and carpet textures. The OUTEX -13 , OUTEX -14, OUTEX -20 and OUTEX -30 databases have 68 classes having samples of canvas, cardboard, carpet, foam, paper, rubber, tile, granite, sandpaper, wood and barley rice textures. Fig. 3 shows sample texture images from each class of OUTEX - 10 database and Fig. 4 shows the samples from each major class of OUTEX 13 database.

\section{B. Performance evaluation metrics}

To evaluate the texture classification performance, the following metrics are employed which are defined in terms of true positive (TP), true negative (TN), false positive (FP) and false negative $(\mathrm{FN})$. These metrics are calculated excluding the inconclusive results.

$$
\begin{gathered}
\text { Accuracy }=\frac{T P+T N}{T P+T N+F P+F N} \\
\text { ErrorRate }=\frac{F P+F N}{T P+T N+F P+F N} \\
\text { Sensitivity }=\frac{T P}{T P+F N} \\
\text { Specificity }=\frac{T N}{T N+F P}
\end{gathered}
$$

C. Experimental setup and Procedure 
The experiments are carried out in MATLAB 2013 environment with the system specification of Inter Core i7 Processor $(4.2 \mathrm{GHz})$ with $8 \mathrm{~GB}$ RAM on Windows 7 64-bit operating system. In this work, we use nearest neighbor classifier with Chi-squared based distance measure.

The experiments are carried out with the following procedure. The database is read and by applying various descriptors discussed in Section 2, the features are extracted using LBP, ULBP.u2 (LBP with uniform patterns), ULBP.ri (LBP with rotation invariance), ULBP (LBP with uniform and rotation invariance), LBPHF, RLBP, LBPV and DRLBP feature descriptors. These features are used to build the classification model. The classification performance is evaluated in terms of classification accuracy, error rate, sensitivity and specificity.
The performance evaluation of LBP family descriptors namely LBP, ULBP.u2, ULBP.ri, ULBP, LBPHF, RLBP, LBPV and DRLBP for texture classification is carried out with two experiments. In Experiment 1, we fix the parameters $\mathrm{R}=1$ and $\mathrm{P}=8$, and extract features using all the eight feature descriptors. The classification accuracy of these eight feature descriptors with nearest neighbor classifier is shown in Fig. 5. From Fig. 5, it is observed that the descriptors with rotation invariance perform better for databases with texture samples at various rotation angles. The radar plot shown in Fig. 6 shows the error rate of descriptors on different databases. The error rate is high for the OUTEX-TC-14 database except for LBP descriptor. The sensitivity and specificity for each case in Experiment 1 is listed in Table 2. These parameters are calculated omitting the inconclusive results.

\section{Results and Discussion}

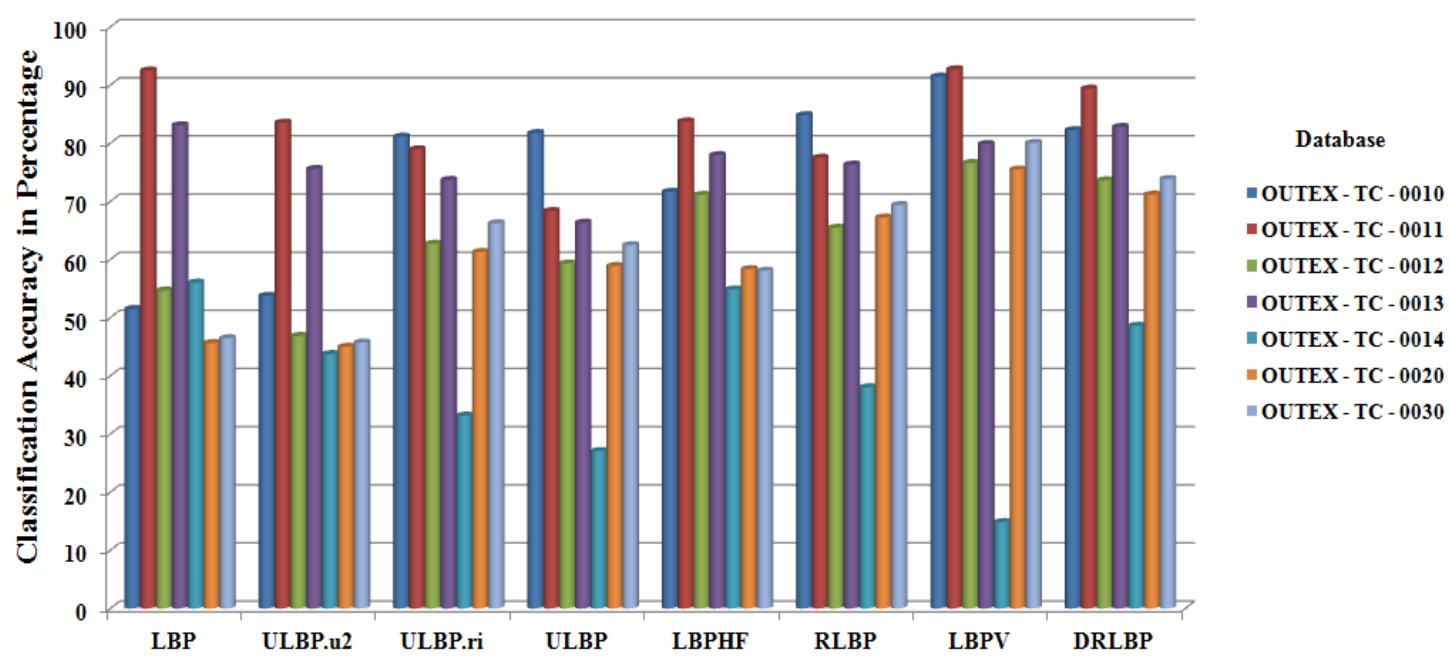

Fig.5. Classification accuracy (in percentage) of LBP family descriptors on various texture databases

\section{Classification Error Rate}

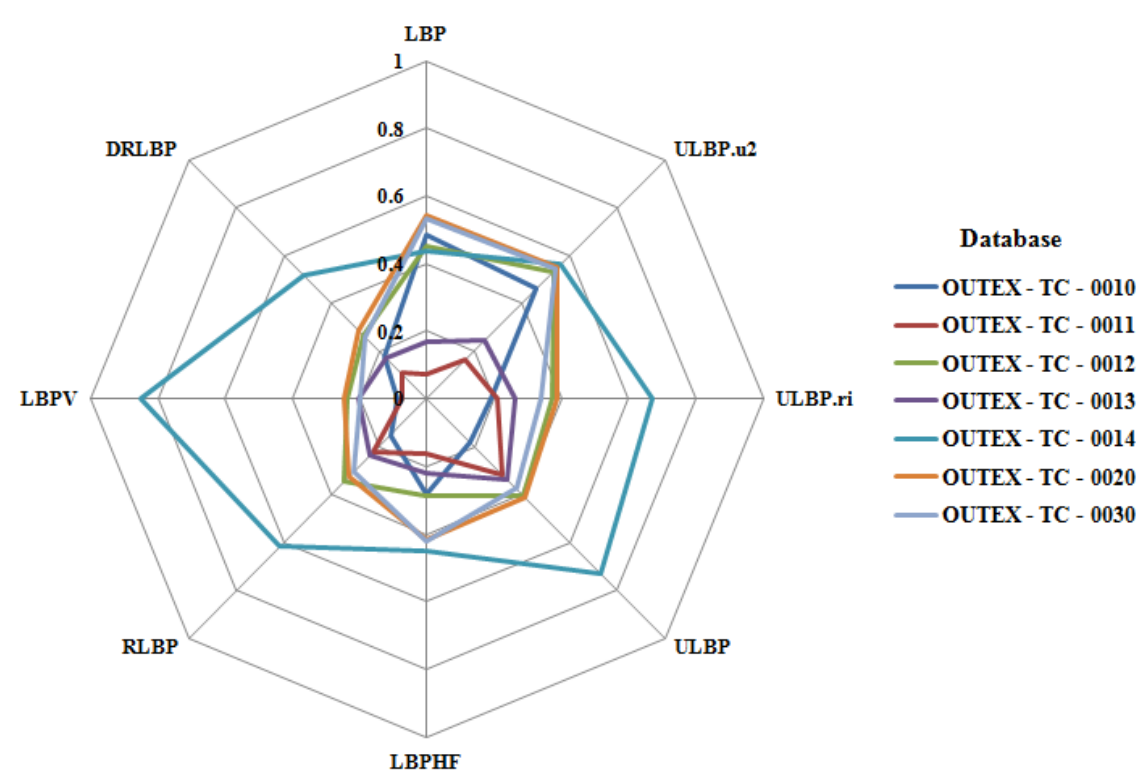

Fig.6. Classification error rate of LBP family descriptors on various texture databases 
Table 2. Comparison of Sensitivity and Specificity of LBP family descriptors on various texture databases

\begin{tabular}{|c|c|c|c|c|c|c|c|c|}
\hline \multirow{2}{*}{$\begin{array}{l}\text { Sensitivity/ } \\
\text { Specificity }\end{array}$} & \multirow[b]{2}{*}{ Descriptor } & \multicolumn{7}{|c|}{ OUTEX } \\
\hline & & $\begin{array}{l}\text { TC - } \\
0010\end{array}$ & $\begin{array}{l}\text { TC - } \\
0011\end{array}$ & $\begin{array}{l}\text { TC - } \\
0012\end{array}$ & $\begin{array}{l}\text { TC - } \\
0013\end{array}$ & $\begin{array}{l}\text { TC - } \\
0014\end{array}$ & $\begin{array}{l}\text { TC - } \\
0020\end{array}$ & $\begin{array}{l}\text { TC - } \\
0030\end{array}$ \\
\hline \multirow{8}{*}{ Sensitivity } & LBP & 0.625 & 1.000 & 0.683 & 1.000 & 0.950 & 0.519 & 0.512 \\
\hline & ULBP.u2 & 0.468 & 0.950 & 0.394 & 1.000 & 0.450 & 0.394 & 0.400 \\
\hline & ULBP.ri & 0.625 & 1.000 & 0.439 & 1.000 & 0.500 & 0.375 & 0.569 \\
\hline & ULBP & 0.737 & 0.800 & 0.489 & 1.000 & 0.500 & 0.444 & 0.656 \\
\hline & LBPHF & 0.681 & 1.000 & 0.694 & 1.000 & 0.650 & 0.619 & 0.512 \\
\hline & RLBP & 1.000 & 0.950 & 0.872 & 1.000 & 0.500 & 1.000 & 1.000 \\
\hline & LBPV & 0.975 & 0.950 & 0.455 & 1.000 & 0.400 & 0.931 & 0.975 \\
\hline & DRLBP & 0.981 & 0.900 & 0.638 & 1.000 & 0.850 & 0.806 & 0.800 \\
\hline \multirow{8}{*}{ Specificity } & LBP & 0.989 & 0.987 & 0.997 & 1.000 & 1.000 & 0.997 & 0.992 \\
\hline & ULBP.u2 & 1.000 & 1.000 & 0.999 & 1.000 & 1.000 & 0.999 & 0.997 \\
\hline & ULBP.ri & 0.999 & 0.976 & 0.999 & 1.000 & 0.997 & 0.999 & 0.999 \\
\hline & ULBP & 1.000 & 0.963 & 0.999 & 1.000 & 0.998 & 0.999 & 0.997 \\
\hline & LBPHF & 0.994 & 0.976 & 0.994 & 1.000 & 1.000 & 0.999 & 0.998 \\
\hline & RLBP & 0.999 & 1.000 & 1.000 & 1.000 & 0.999 & 0.999 & 0.999 \\
\hline & LBPV & 0.999 & 1.000 & 0.992 & 1.000 & 0.998 & 0.999 & 0.999 \\
\hline & DRLBP & 1.000 & 1.000 & 0.9990 & 1.000 & 1.000 & 0.999 & 0.998 \\
\hline
\end{tabular}

In Experiment 2, we repeat the same procedure as in Experiment 1 with different values of $\mathrm{R}$ and $\mathrm{P}$ (Fig. 7). For simplicity, we use only OUTEX TC - 0010 database for this experiment. It is interesting to find that the simple LBP operator has no effect on $\mathrm{R}$ and $\mathrm{P}$ changes. On the other hand, the maximum classification accuracy is achieved with $\mathrm{R}=3$ and $\mathrm{P}=8$, and DRLBP descriptor achieves the best performance with classification accuracy over $99 \%$.

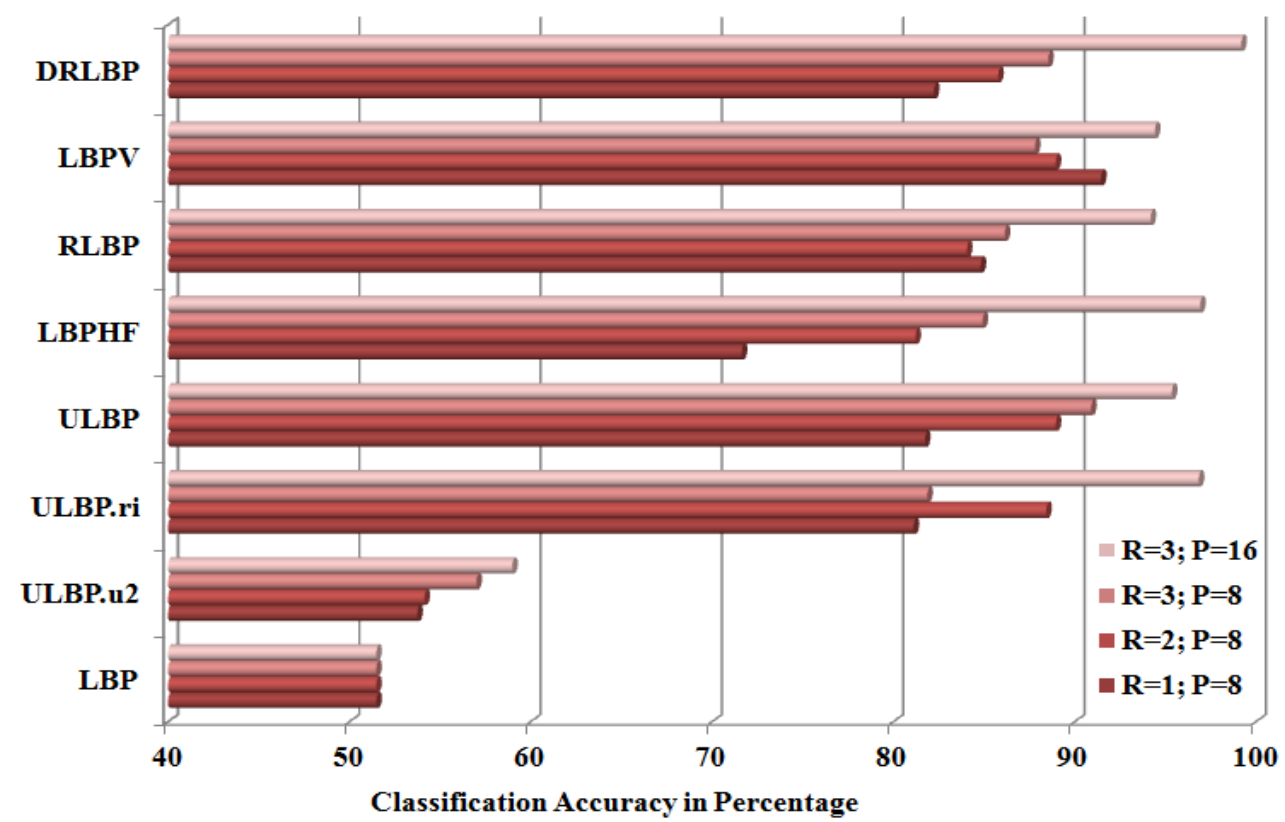

Fig.7. Classification accuracy (in percentage) of LBP family descriptors on OUTEX TC -0010 database for different R and P values 


\section{CONCLUSION}

LBP family descriptors are simple yet efficient and hence used in many pattern recognition applications such as face recognition, object recognition, etc. This paper presented the performance evaluation of LBP family descriptors namely LBP, ULBP.u2, ULBP.ri, ULBP, LBPHF, RLBP, LBPV and DRLBP for texture classification. Two experiments are done to evaluate the performance of these descriptors on OUTEX texture databases. We observed that the LBP family descriptors with rotation invariance capability exhibit better texture classification performance. We also tested these descriptors with different LBP parameters such as radius and neighborhood.

\section{REFERENCES}

[1] Materka, Andrzej, and Michal Strzelecki. "Texture analysis methods-a review." Technical university of lodz, institute of electronics, COST B11 report, Brussels (1998). 9-11.

[2] Ahonen, Timo, Abdenour Hadid, and Matti Pietikainen. "Face description with local binary patterns: Application to face recognition." IEEE transactions on pattern analysis and machine intelligence 28.12 (2006): 20372041.

[3] Nguyen, Duc Thanh, Zhimin Zong, Philip Ogunbona, and Wanqing Li. "Object detection using non-redundant local binary patterns." In Image Processing (ICIP), 2010 17th IEEE International Conference on, pp. 4609-4612. IEEE, 2010.

[4] Trefný, Jirí, and Jirí Matas. "Extended set of local binary patterns for rapid object detection." In Computer Vision Winter Workshop, pp. 1-7. 2010.

[5] Heikkilä, M., Pietikäinen, M., \& Schmid, C. (2009). Description of interest regions with local binary patterns. Pattern recognition, 42(3), 425-436.

[6] Zhu, Chao, Charles-Edmond Bichot, and Liming Chen. "Multi-scale color local binary patterns for visual object classes recognition." In Pattern Recognition (ICPR), 2010 20th International Conference on, pp. 3065-3068. IEEE, 2010.

[7] Liao, W. H., \& Young, T. J. (2010, December). Texture classification using uniform extended local ternary patterns. In Multimedia (ISM), 2010 IEEE International Symposium on (pp. 191-195). IEEE.

[8] Ahonen, T., Matas, J., He, C., \& Pietikäinen, M. (2009). Rotation invariant image description with local binary pattern histogram fourier features. Image analysis, 61-70.

[9] Khellah, F. M. (2011). Texture classification using dominant neighborhood structure. IEEE Transactions on Image Processing, 20(11), 3270-3279.

[10] http://www.outex.oulu.fi/index.php?page=classification [Accessed on 22nd May 2017]

[11] Ojala, T., Pietikäinen, M., \& Mäenpää, T. (2001, March). A generalized local binary pattern operator for multiresolution gray scale and rotation invariant texture classification. In International Conference on Advances in Pattern Recognition (pp. 399-408). Springer Berlin Heidelberg.

[12] T. Maenpaa, T. Ojala, and M. Pietikainen, "Robust texture classification by subsets of Local Binary Patterns," in Proceedings of the 15th International Conference on Pattern Recognition, vol. 3, pp. 947-950, Barcelona, Spain, 2000.

[13] Guo, Z., Zhang, L., \& Zhang, D. (2010). Rotation invariant texture classification using LBP variance (LBPV) with global matching. Pattern recognition, 43(3), 706-719.

[14] R.Mehta,K.Egiazarian, Rotated local binary pattern (RLBP): Rotation invariant tex-ture descriptor, Proceedings of International Conference on Pattern Recognition Applications and Methods (2013) 497-502.

[15] Mehta, R., \& Egiazarian, K. (2016). Dominant rotated local binary patterns (DRLBP) for texture classification. Pattern Recognition Letters, 71, 16-22.

\section{Authors' Profiles}

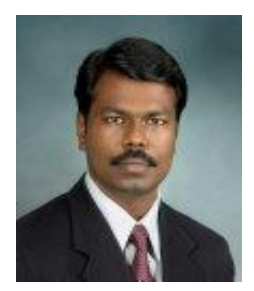

Dr. D. Asir Antony Gnana Singh received the Bachelor of Engineering in Computer Science and Engineering, Master of Engineering in Computer Science and Engineering, Master of Business Administrator in Human Resource Management, and Ph. D in Information and Communication Engineering degrees from Anna University, India. He is currently working as a teaching fellow in the Department of Computer Science and Engineering, Anna University, BIT-Campus, Tiruchirappalli, India. His research interests include data mining, wireless networks, parallel computing, mobile computing, computer networks, image processing, software engineering, soft computing, cloud computing, big data analytics, teaching learning process and engineering education, human resource management.

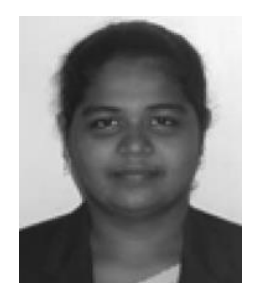

Dr. E. Jebamalar Leavline received the Ph.D, M. Eng. and B. Eng. degrees from Anna University, India, and received the MBA degree from Alagappa University, India. She is currently working as an assistant professor in the Department of Electronics and Communication Engineering, Bharathidasan Institute of Technology, Anna University, India. Her research interests include image processing, signal processing, VLSI design, data mining, teaching learning process and engineering education.

P. Maheswari is working as assistant professor in Department of ECE in K.Ramakrishnan College of Technology, Samayapuram, Tiruchirappalli, India. Her research interests include image processing and signal processing.

How to cite this paper: E. Jebamalar Leavline, D. Asir Antony Gnana Singh, P. Maheswari, " Local Binary Pattern Family Descriptors for Texture Classification", International Journal of Image, Graphics and Signal Processing(IJIGSP), Vol.10, No.10, pp. 40-45, 2018.DOI: 10.5815/ijigsp.2018.10.04 